\title{
Simulation Analysis of Ad-Hoc On-Demand Distance Vector Routing Protocol Performance in Mobile Ad Hoc Network Using Taguchi Design Approach
}

\author{
Hazura Mohamed ${ }^{a}$, Muhammad Hisyam Lee ${ }^{b, *}$, Mazalan Sarahintu ${ }^{b}$, Shaharuddin Salleh ${ }^{b}$ and Bahrom \\ Sanugi ${ }^{c}$ \\ ${ }^{a}$ Department of Industrial Computing, Faculty of Information Science and Technology, Universiti Kebangsaan Malaysia, 43600 \\ Bangi, Selangor, Malaysia.

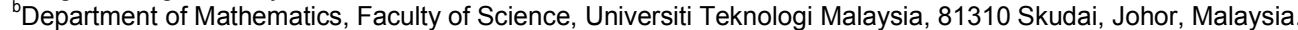 \\ ${ }^{c}$ Faculty of Science and Technology, Universiti Sains Islam Malaysia, 78100 Nilai, Negeri Sembilan, Malaysia. \\ *To whom correspondence should be addressed. E-mail: mhl@utm.my
}

Received: 31 October 2008

http://dx.doi.org/10.11113/mjfas.v4n2.47

\section{ABSTRACT}

Mobile ad-hoc network routing protocol performance is evaluated mostly through simulative studies. One-factor-at-atime approach is commonly used to quantify the effect of various factors on the protocol performance. In this work, the effect of several factors was investigated simultaneously and speedily with the application of Taguchi design of experiments. The effect of the factors which include terrain size, node speed, network size, transmission range, transmission rates, pause time, and maximum connection on routing overhead was quantified. Analysis signal-to-noise ratio was performed to determine the best possible combination of factors and analysis of variance (ANOVA) was carried out to identify the significant factors that affect the routing overhead. The results showed that network size, transmission range and maximum connection play a significant role in minimizing routing overhead. The results were confirmed experimentally at $95 \%$ confidence interval.

| Mobile ad-hoc network | AODV | Taguchi experimental design | ANOVA |

\section{Introduction}

Mobile ad hoc networks (MANETs) are collection of wireless nodes communicating among themselves over wireless link without the help of any network infrastructure such base station or access point [11]. Routing is a fundamental problem in such a network. Each nodes are responsible for dynamically discovering other nodes that can communicate directly. A key assumption is not all nodes can directly communicate with each other because of limited transmission range. Therefore, nodes also as routers are required to relay packets on behalf of other nodes in order to deliver data across the network.

Numerous routing protocols have been proposed in the literature to solve the routing problem in MANETs. Basically, these protocols may be categorized as table-driven and on-demand, according to the way the mobile nodes exchange routing information. The table-driven protocols, such as Destination Sequence Distance Vector 
(DSDV) [2], periodically disseminate routing information among all the nodes in the network, so that every host has the up-to-date information for all possible routes [9]. On-demand routing protocols, such as Ad hoc Ondemand Distance Vector (AODV) [1] and Dynamic Source Routing (DSR) [3], operate on a need basis, discover and maintain only active route that currently used for delivering data packets [9].

Here, we briefly describe the AODV protocol that we investigate. The AODV routing protocol is one of the most popular in on-demand routing protocols in MANETs. It has the ability to quickly adapt to dynamic link condition with low processing and memory overhead [16]. AODV offers low network utilization and uses destination sequence number to guarantee loop freedom. In AODV, requests for route discovery are initiated only when sources need them, something that reduces drastically the packet routing overhead, and hence the overall packet delays [6]. In addition, "bad-news", concerning link failures, travel back to sources quite quickly, so as for the new route discovery procedure to be initiated soon after the failure. This makes AODV very useful in variety of applications, such as in battlefields, emergency services and video conferences, especially in large node population [6].

There is a lot of work done on evaluating performance of routing protocols in MANETs [6], [9], [13], [16] and [18] but most of the work is limited on identifying the impact of factors on one or more performance metrics using one-factor-at-a-time approach. Our work differs in that we use Taguchi design of experiment approach to investigate the performance of the AODV protocol. Our study has shown that Taguchi method is a powerful technique in quantifying the effect of several factors simultaneously such as terrain, node speed, network size, transmission range, and transmission rates, pause time and maximum connection on the routing overhead. Author [8] has been initially created the use of Taguchi method in determine the effects of influential factors on MANETs routing protocol performance.

\section{Taguchi Design Approach}

Taguchi design approach (TDA) also known as a robust parameter design has been successfully applied in many areas of engineering, biological science and including physical science to compare the effects of multiple factors, together with their interactions simultaneously. In comparison with a traditional full factorial design of experiments, TDA provide a significant reduction in the size of experiment, thereby speeding up the experimental process [15].

Two important tools used in TDA are orthogonal arrays (OAs) and signal-to-noise ratio (SNR). OAs allows researchers to study many combination factors simultaneously and can be used to estimate the effects of each factor. The SNR is the quality indicator by which one can evaluate the effect of changing a particular factor on the performance of the process.

According to the TDA, the SNR is the ratio of signal to noise where signal represent the desirable value, and noise represent the undesirable value. There are three types of quality characteristics in the TDA; lower-the-better (LB), nominal-the-best (NB), and higher-the-better (LB) [5]. The objectives of this study is to minimize routing overhead in message delivery process. Routing overhead was calculated by taken the total number of packet routing. Therefore, performance characteristic of lower-the-better for routing overhead was implemented in this study and the formulation of SNR for routing overhead can be written as [5]:

$$
\eta_{\mathrm{RO}}=10 \log \frac{1}{\mathrm{r}} \sum_{\mathrm{i}=1}^{\mathrm{r}} \mathrm{y}_{\mathrm{i}}^{2}
$$

where $\eta_{R O}$ is the SNR of routing overhead, $r$ is the number of simulation repetitions under the same experimental number and $y$ is the routing overhead for each experiment number. 
In TDA, the appropriate orthogonal array for the simulation experiment depends on the number of factors and the number of factor levels. The degree of freedom for the orthogonal array should be greater or equal to those for the factors under study. Therefore, to select a suitable OA for an experiment, the following inequality must be satisfied [10].

$$
\mathrm{Df}_{\alpha} \geqslant \sum \mathrm{Df}
$$

where $\sum_{\mathrm{Df}}$ is the total degree of freedom of all factors considered in experiment, $\mathrm{Df}_{\alpha}$ is the total degree of freedom available in $\mathrm{OA}$.

\section{Simulation Environment}

Our goal of this work is to investigate the impact of terrain, node speed, network size, transmission range, and transmission rates, pause time and maximum connection on the performance of AODV protocol. We utilized two different value levels for each factor. Generally, two levels of factors well in screening experiments [4] in identifying which factors have large effects to response. Each node is placed randomly and freely to move and communicate in the terrain, which is an area of network. After remaining at the location for the specified pause time, the nodes then move to the new location at a speed uniformly chosen between a minimum and a maximum speed (meter/sec). The transmission range of each node was set as $30 \mathrm{~m}$ and $100 \mathrm{~m}$, as considered a WiFi's range [7]. In this simulation study we used constant bit rate CBR sources that continuously transmit 1024-byte data packet at a certain value of sending packet rates. The packet rates were chosen in order to have a small and moderate congestion in the network. These factors were based on the previous study [10] but the differences are routing protocol evaluated and scenario simulation. These factors were selected because from literature, they are important factors that have potential to effect the performance of routing protocols and always used in their evaluating performance of routing protocol in MANETs. Table 1 shows the factors selected and their level values.

The goal of our simulation experiments is to examine and quantify the affects of those seven factors on the performance of AODV routing protocol. To achieve this goal, we used the $\mathrm{L}_{8}\left(2^{7}\right)$ orthogonal array in our experiment. Our simulation experiment was conducted using network simulator, ns-2.29 [19]. We use ns-2 because it has been used in various works in the literature [17] and due to the fact that AODV is already implemented in the simulator. The simulation of the mobile network has been carried out on an Intel ${ }^{\circledR}$ Core $^{\mathrm{TM}} 2$ Duo CPU processor at $2.33 \mathrm{GHz}, 2046 \mathrm{MB}$ of RAM and running Linux Fedora Core 3. Each simulation was executed for 900 second.

Table 1: Selected factors and levels

\begin{tabular}{|clcc|}
\hline \multirow{2}{*}{ Label } & \multirow{2}{*}{ Factor } & \multicolumn{2}{c|}{ Level } \\
& & 1 & 2 \\
\hline A & Terrain size $\left(\mathrm{m}^{2}\right)$ & $200 \times 200$ & $100 \times 400$ \\
B & Node speed $\left(\mathrm{ms}^{-1}\right)$ & 0.72 & 1.34 \\
C & Network size & 50 & 100 \\
D & Transmission range (m) & 30 & 100 \\
E & Transmission rates (packets/s) & 1 & 10 \\
F & Pause time (s) & 60 & 200 \\
G & Maximum connection & 2 & 8 \\
\hline
\end{tabular}




\section{Results and Discussion}

In this work, the lower routing overhead is the indication of better performance. Simulation experiments were replicated eights and randomized to minimize the bias from both experiments and within experiment error.

\subsection{Analysis of the signal-to-noise ratio}

The SNRs computed using Eq (1) for routing overhead for each of the 8 experiments are shown in Table 2. The greater SNR value of each performance characteristic corresponds to a better performance.

Table 2: Experimental design using the $\mathrm{L}_{8}$ and $\mathrm{SNR}$ computed

\begin{tabular}{|c|ccccccc|c|}
\hline \multirow{2}{*}{$\begin{array}{c}\text { Experiment } \\
\text { number }\end{array}$} & $\mathrm{A}$ & $\mathrm{B}$ & $\mathrm{C}$ & $\mathrm{D}$ & $\mathrm{E}$ & $\mathrm{F}$ & $\mathrm{G}$ & $\begin{array}{c}\text { SNR for } \\
\text { routing } \\
\text { overhead }\end{array}$ \\
\cline { 2 - 6 } & 1 & 1 & 1 & 1 & 1 & 1 & 1 & -76.009 \\
2 & 1 & 1 & 1 & 2 & 2 & 2 & 2 & -64.793 \\
3 & 1 & 2 & 2 & 1 & 1 & 2 & 2 & -91.114 \\
4 & 1 & 2 & 2 & 2 & 2 & 1 & 1 & -65.458 \\
5 & 2 & 1 & 2 & 1 & 2 & 1 & 2 & -97.420 \\
6 & 2 & 1 & 2 & 2 & 1 & 2 & 1 & -65.403 \\
7 & 2 & 2 & 1 & 1 & 2 & 2 & 1 & -72.031 \\
8 & 2 & 2 & 1 & 2 & 1 & 1 & 2 & -71.277 \\
\hline
\end{tabular}

The average SNRs for each level of each factor for rouying overhead are shown in Table 3 . The absolute difference between the average SNR values of the two levels (denoted as delta) reveals the effect of each factor and the values are also given in Table 3. Basically, the larger the difference, the stronger the factor influence the performance characteristic. By identifying the order of the effects from largest to smallest for routing overhead from SNR response table (see Table 3), factor D is the strongest effect. Factor G is the second and followed by the factor $\mathrm{C}$. Since the difference between the effect of factor $\mathrm{C}$ and the effect of factor $\mathrm{F}$ was greater ( 4.615$)$ than the difference between the effect of factor $\mathrm{G}$ and the effect of factor $\mathrm{C}$ ( 2.604 ), it was ruled the logical breaking point [5]. Therefore, the strong effects and their recommended levels are C1, D2, and G1. Due to the mild effects, the preferred levels for factor A, B, E, and F based on highest SNR values in Table 3.

Table 3: Average SNR for routing overhead

\begin{tabular}{|cccc|}
\hline Factor & Level 1 & Level 2 & Delta \\
\hline A & -74.343 & -76.533 & 2.190 \\
B & -75.906 & -74.969 & 0.937 \\
C & -71.027 & -79.849 & 8.822 \\
D & -84.143 & -66.733 & 17.410 \\
E & -75.951 & -74.925 & 1.026 \\
F & -77.541 & -73.335 & 4.206 \\
G & -69.725 & -81.151 & 11.426 \\
\hline
\end{tabular}




\subsection{Analysis of Variance (ANOVA)}

The purpose of the ANOVA is to investigate which factors significantly affect the performance characteristic. ANOVA helps in formally testing the significance of all factors by comparing the mean square against an estimate of the experimental errors at specific confidence level. The relative effect of the different factor can be obtained by separating the total variation of the SNR, which is measured by the sum of the squared deviations from the total mean SNR, into contributions by each of factors and the error. The total sum of squared deviations SST from the total mean SNR $\eta_{m}$ can be calculated as [15]:

$$
\mathrm{SS}_{\mathrm{T}}=\sum_{\mathrm{i}}^{\mathrm{n}}\left(\eta_{\mathrm{i}} \quad \eta_{\mathrm{m}}\right)^{2}
$$

where $n$ is the number of experiments in the orthogonal array and $\eta_{i}$ is the mean SNR for the $i$ th experiment. The percentage contribution by each factor in the total sum of the squared deviations can be used to evaluate the importance of the factor on the performance characteristic. The percentage contribution $P$ of each factor can be calculated as [15]:

$$
\mathrm{P}=\frac{\mathrm{SS}_{\mathrm{d}}}{\mathrm{SS}_{\mathrm{T}}}
$$

where $S S_{d}$ is the sum of the squared deviations, which is defined as the sum of squares of factors minus the error variance times the degree of freedom of each factor. The greater the percentage contribution, the greater the influence a factor has on the performance. In addition, the F-test named after Fisher [14] can also be used to determine which factors have significant effect on the performance characteristic. Usually when the $F$-value is large, the factor has a significant effect on the performance characteristic. Table 4 presents the results of ANOVA for routing overhead. Since the ANOVA has resulted in zero degree of freedom (df) for error term, it is necessary to pool the factor if its influence is $10 \%$ or lower than the most influential factor [15] for correct interpretation of results. According to Table 4, at 95\% confidence level shows that transmission range D (55.43\%), maximum connection G (23.22\%) and network size C (13.38\%) are statistically significant effect on the routing overhead.

Table 4: ANOVA table for routing overhead

\begin{tabular}{|cccccc|}
\hline Significant factors & df & $\begin{array}{c}\text { Sum of Squares } \\
(\mathrm{SS})\end{array}$ & $\begin{array}{c}\text { Mean square } \\
(\mathrm{MS})\end{array}$ & $F$-ratio & $P$ \\
\hline $\mathrm{C}$ & 1 & 155.634 & 155.634 & $12.752^{*}$ & 13.38 \\
$\mathrm{D}$ & 1 & 606.281 & 606.281 & $49.675^{*}$ & 55.43 \\
$\mathrm{G}$ & 1 & 261.094 & 261.094 & $21.392^{*}$ & 23.22 \\
Pooled error & 4 & 48.820 & 12.205 & & 7.97 \\
& & & & \\
Total & 7 & 1071.829 & & & \\
& & & & & \\
\end{tabular}

The optimum value of routing overhead can predict at the selected level of significant factors. The average effects of the factors shown in Table 3, indicated that level 1 of both network size and maximum connection and level 2 of transmission range will be included in optimum condition. The estimated SNR using the optimal level of the significant factors for the routing overhead could be computed as follows [14]: 


$$
\begin{aligned}
\eta_{\text {predicted }} & =C_{1}+D_{2}+G_{1} \quad 2 \bar{\eta} \\
& =72.027 \quad 66.733 \quad 69.725+150.876 \\
& =56.609
\end{aligned}
$$

Because Eq (5) is a point estimation calculated by using experimental data to determine whether the result of confirmation experiment are meaningful or not, the confidence interval must be evaluated. The confidence interval at at $95 \%$ confidence level, may calculated as follows [14]:

$$
\eta_{\text {predicted }} \pm \sqrt{\mathrm{F}_{0.5,1, \mathrm{fe}} \times \mathrm{V}_{\mathrm{e}} \times \frac{1}{\mathrm{n}_{\text {eff }}}+\frac{1}{\mathrm{r}}}
$$

where fe is error degree of freedom, $V_{e}$ is the variance of the error term, $r$ is the number of repetition and $n_{e f f}$ is given by

$$
\mathrm{n}_{\mathrm{eff}}=\frac{\text { total number of SNR }}{1+\begin{array}{l}
\text { total degree of freedom } \\
\text { of the significant factor }
\end{array}}
$$

Table 5 shows the comparison of the expected SNR routing overhead at $95 \%$ confidence level with the measured SNR value of confirmation experiment. Eight confirmation runs of best factor level combinations $\left(\mathrm{A}_{1} \mathrm{~B}_{2} \mathrm{C}_{1} \mathrm{D}_{2} \mathrm{E}_{2} \mathrm{~F}_{2} \mathrm{G}_{1}\right)$ were carried out. Good agreement between the expected and actual SNR of routing overhead is observed.

Table 5: Result of confirmation experiment for routing overhead

\begin{tabular}{|l|ccc|}
\hline \multirow{4}{*}{} & \multicolumn{3}{|c|}{$\begin{array}{c}\text { Best factor-level } \\
\text { combinations }\end{array}$} \\
\cline { 2 - 4 } & \multicolumn{3}{|c|}{$\mathrm{A}_{1} \mathrm{~B}_{2} \mathrm{C}_{1} \mathrm{D}_{2} \mathrm{E}_{2} \mathrm{~F}_{2} \mathrm{G}_{1}$} \\
\cline { 2 - 4 } & Predicted & Confidence interval & Experimented \\
SNR & -56.609 & $(-63.648,-49.750)$ & -56.269 \\
\hline
\end{tabular}

\section{Conclusion}

The use of the Taguchi experimental design approach to determine the effects of factors on the AODV protocol performance with regards to routing overhead has been reported in this paper. The selected factors were taking into consideration of the seven factors that always used in evaluating performance of routing protocols. The $\mathrm{L}_{8}$ orthogonal array was utilized in the simulation studies under varying terrain sizes, node speeds, network sizes, transmission ranges, transmission rates, pause times, and maximum connections using ns-2 simulator were conducted. 
From the analysis of the results using signal-to-noise ratio, analysis of variance and Taguchi's optimization method, the following can be concluded from the present study:

- Statistical results (at 95\% confidence level) show that the transmission range is the major effect on routing overhead by $55.43 \%$, followed by maximum connection $(23.22 \%)$ and network size $(13.82 \%)$.

- The best combination values of factors were confirmed with verification experiments and the results are fall within the $95 \%$ confidence interval of routing overhead.

Since the selection of value level of factors is sensitive, it is important to take note that all the findings in this paper were totally based upon the factor levels considered in this study and may vary if different factor levels were used.

\section{Acknowledgement}

This work was supported by University of Technology Malaysia (UTM) under Grant Vote No. 79220 and by Ministry of Science, Technology and Innovation (MOSTI), Malaysia, under Grant 01-01-06-SF0234.

\section{References}

[1] C. Perkins, E. Royer, S. Das. Ad Hoc On-Demand Distance Vector (AODV) Routing. RFC 3561, InternetDraft, draft-ietf-manet-aodv-11.txt. 2003.

[2] C. Perkins and P. Bhagwat. Highly Dynamic Destination-Sequenced Distance-Vector Routing (DSDV) for Mobile Computers. SIGCOMM Comput. Commun. Rev. 24, 4 (Oct. 1994), 234-244.

[3] D. B. Johnson, D. A. Maltz, Hu, Y-C. and Jetcheva, J. G. The Dynamic Source Routing Protocol for Mobile Ad Hoc Networks (DSR). Internet-drafts. 2004.

[4] D. C. Montgomery. Design and Analysis of Experiments. 6th ed. Hoboken, N.J.:John Wiley \& Sons, Inc., 2004.

[5] G.S. Peace. Taguchi Method: A Hands-on Approach. Reading, Mass.: Addison-Wesley Publishing Company. 1992.

[6] I. Boustis, G. Jakllari, T. Repantis, and M. Molle. A Performance Comparison of Routing Protocols for Large-Scale Wireless Mobile Ad Hoc Networks. Web Protocols White Papers, 2006.

[7] J. McLure and J.Reno. Keeping Up With Wi-Fi. Newsweek: Monday, November 24, 2003.

[8] M. H. Lee. Formulation of Properties for Motion Prediction in Wireless Networks and Simulation Analysis of Dynamic Destination-Sequenced Distance-Vector Protocol. Ph.D Thesis. Universiti Teknologi Malaysia, 2003.

[9] M. Lakshmi, and P.E. Sankaranarayanan. Performance Analysis of Three Routing Protocols in Mobile Ad Hoc Wireless Networks. Asian Journal of Information Technology. Vol. 4 (2005) 1195-1200.

[10] M. Sarahintu, M.H. Lee and H. Mohamed. Determining the Effects of Scenario Metrics on the Performance of Dynamic Source Routing using Taguchi Approach. Matematika. Vol. 23:2(2007) 121-132.

[11] M. S.Corson, J. P.Macker, and G. H. Cirincione. Internet-Based Mobile Ad Hoc Networking. IEEE Internet Computing 3, 4 (Jul. 1999) 63-70.

[12] M. W. Totaro, and D. D. Perkins. Using statistical design of experiments for analyzing mobile ad hoc networks. Proceedings of the 8th ACM international symposium on Modeling, analysis and simulation of wireless and mobile systems. October 10-13. Montreal, Quebec: ACM, 2005, 159-168.

[13] P. Johansson, T. Larsson, and N. Hedman. Senario-based Performance Analysis of Routing Protocols for Mobile Ad Hoc Networks. Proc. Of $5^{\text {th }}$ Annual ACM/IEEE International Conference on Mobile Computing and Networking, Seattle, 1999.

[14] P.J. Ross. Taguchi Techniques for Quality Engineering. $2^{\text {nd }}$ ed., McGraw-Hill Inc., New York, 1996. 
[15] R.K.Roy, Design of Experiment Using the Taguchi Approach: 16 Steps to Product and Process Improvement, Wiley, New York, 2001.

[16] S. Ahmed and .S. Alam. Performance Evaluation of Important Ad Hoc Network Protocols. EURASIP Journal on Wireless Communications and Networking. Vol.2006 (2005) 1-11.

[17] S.Kurkowski, T.Camp, and M.Colagrosso. . MANET simulation studies: The incredibles. ACM's Mobile Computing and Communications Review, 9:4 (2005) 50-61.

[18] S.R. Das, C.E. Perkins and E.M. Royer. Performance Comparison of Two On-demand Routing Protocols for Ad hoc Networks. In Proceedings of the IEEE Conference on Computer Communications (INFOCOM), 2000)\.

[19] The network simulator ns-2. http://www.isi.edu/ns/nam/ns/. 